\title{
Lubricated friction on nanopatterned surfaces via molecular dynamics simulations
}

\author{
R. Capozza, ${ }^{1}$ A. Fasolino, ${ }^{2}$ M. Ferrario, ${ }^{1}$ and A. Vanossi ${ }^{1}$ \\ ${ }^{1}$ CNR-INFM National Research Center S3 and Department of Physics, University of Modena and Reggio Emilia, Via Campi 213/A, \\ 41100 Modena, Italy \\ ${ }^{2}$ Institute for Molecules and Materials, Radboud University Nijmegen, Heyendaalseweg 135, 6525 AJ Nijmegen, The Netherlands
}

(Received 11 October 2007; revised manuscript received 22 April 2008; published 24 June 2008)

\begin{abstract}
Recent studies have proved the usefulness of macroscopic surface patterning for the improvement of tribological performances of sliding contacts. Here we investigate the effects of scaling down the texturing dimensions to the nanoscale. By means of classical molecular dynamics simulations, we show that the sliding frictional properties of a thin lubricant film are significantly affected by the presence of nanoscale superficial patterning of the moving confining walls, leading to a "mitigation" of the so-called stick-slip regime and to a consequent reduction of friction. We believe these findings to be relevant for nanotechnology applications.
\end{abstract}

DOI: $10.1103 /$ PhysRevB.77.235432

PACS number(s): 81.40.Pq, 46.55.+d, 68.35.Af, 68.08.-p

\section{INTRODUCTION}

Due to continuing device miniaturization, friction imposes serious constraints and limitations on the performance and lifetime of advanced technological microdevices. Durable low-friction surfaces and suitable liquid or solid lubricants are increasingly in demand in hi-tech applications such as magnetic storage systems, microelectromechanical devices (mems), and aerospace components. The difficulty of tribological problems is related to their complexity, namely dealing with systems under a strict size confinement that leaves very limited access to the interface itself.

Introduction of specific microtextures onto a sliding surface, comprising smooth, flat regions interrupted by local depressions, is an often-used approach to improve tribological properties of mechanical sliding contacts. ${ }^{1,2}$ One of the roles of these depressions is to trap wear particles. The elimination of wear debris from the mating interface reduces the ploughing component of friction. Besides, another important function of the surface dimples is to act as reservoir for lubricants capable of feeding the lubricating fluid directly between the two contacting surfaces. ${ }^{3,4}$

Advanced experimental techniques, such as for example focused ion-beam (FIB) milling ${ }^{5,6}$ or electron-beam lithography (EBL), ${ }^{7}$ allow us nowadays to build and control surface structures and morphology with high spatial resolution down to the nanometer scale. This observation raises an interesting question in the context of nanolubrication and friction. Does the tribological response of a sliding interface change in presence of nanoscale textures in boundary lubricated regime?

Numerical molecular dynamics simulations and simplified theoretical approaches have revealed themselves to be extremely useful in giving deeper insights into the nature of friction and tribological processes at the nanoscale. $\mathrm{Nu}-$ merical modeling, even if often idealized, can reproduce most experimental observations such as stick-slip, nature of static friction, dependencies of observations on temperature and other external parameters. But what is even more important and promising is that in many cases predictions made by means of simplified tribological models have been confirmed experimentally. ${ }^{8,9}$ It has recently been shown ${ }^{10}$ how, in presence of textured surfaces, the concerted effect of wetting properties and surface roughness may considerably reduce friction of a thick fluid lubricant past the boundaries. Under a certain pressure $P_{N}$, the microscopic patterning reduces the wettability of the substrate which is only weakly coupled to the liquid lubricant: momentum transfer parallel to the interface is strongly reduced.

When a confined lubricant is characterized by a large surface-to-volume ratio, the surface properties strongly affect its tribological properties. If the width of the lubricant film is reduced to a few atomic layers, e.g., because of the squeezing of the lubricant out of the contact region due to high load when the interface sliding stops, the atoms in the film are ordered into layers parallel to the bounding walls, although this behavior is less pronounced for branched molecules. ${ }^{11,12}$ Both numerical simulations ${ }^{13}$ and experiments ${ }^{14}$ generally conclude that, in this regime of boundary lubrication, the film behaves like a solid (at least in the mechanical sense that static friction is observed), even at temperatures significantly higher than its bulk melting temperature. At low driving relative velocity of the confining walls, the motion in such a system corresponds to the highly dissipative stick-slip dynamics, which is explained by means of the melting-freezing mechanism; ${ }^{13}$ the film melts during slip and solidifies at stick.

In this paper, we investigate the effects of surface nanopatterning on the tribological performances of sliding contacts in the regime of boundary lubrication. The mesoscopic frictional response of the driven top plate is analyzed in terms of the detailed microscopic dynamics and structural rearrangements of the confined lubricant layers under shear. In Sec. II, we present the model used for molecular dynamics simulations, elucidating some computational details. Section III is devoted to show the results of the calculated friction force obtained by varying the model parameters over a wide range of values; the tribological behavior being related to the atomistic dynamics and structure of the embedded lubricant film. In Sec. IV, we examine the role of the pattern density and, finally, we draw conclusions and some perspectives in Sec. V. 


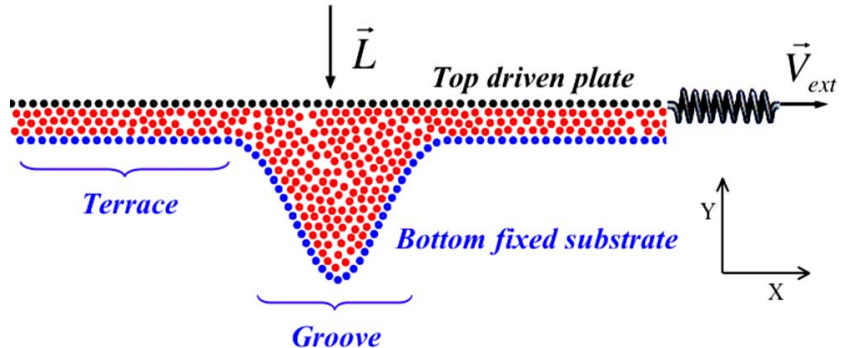

FIG. 1. (Color online) Sketch of the system. The lubricant is confined between the top driven plate and the patterned substrate. Here, the system size in the $x$ direction is 60 ( $L-J$ units)

\section{MODEL AND SIMULATION DETAILS}

We consider here the regime of boundary lubrication, where the sliding surfaces are separated by only a few layers of lubricant molecules, as sketched in Fig. 1. We study a two-dimensional system consisting of a rigid, patterned substrate, a rigid driven top plate and $n_{l}$ lubricant particles confined in between. Our simulations are mainly carried out for the case of three embedded lubricant layers; by increasing the lubricant width (while remaining always in the regime of boundary lubrication), the static friction force needed to initiate the sliding decreases approximately exponentially. ${ }^{15}$ The driven top plate and the bottom substrate are made up of $n_{t}$ and $n_{b}$ particles, respectively, having a constant lattice separation $a_{s}=1$. We use dimensionless units with top, bottom, and lubricant atoms all of mass $m=1$. The top plate of mass $M_{\mathrm{top}}=m \cdot n_{t}$ is pulled through a spring, of elastic constant $K$, connected to a stage which moves along the horizontal $x$ direction with constant velocity $V_{\text {ext }}$. A loading force $\vec{L}$ is applied to the top plate along the negative vertical $y$ direction. Due to the vertical confinement, periodic boundary conditions are applied only along the $x$ direction.

All atoms interact via a 6-12 Lennard-Jones pairwise potential

$$
\begin{gathered}
U^{\alpha \alpha^{\prime}}(r)=U^{\alpha \alpha^{\prime}}\left[\left(\frac{r^{\alpha \alpha^{\prime}}}{r}\right)^{12}-2\left(\frac{r^{\alpha \alpha^{\prime}}}{r}\right)^{6}\right] \\
\alpha, \alpha^{\prime}=l, t, b
\end{gathered}
$$

where $l, t, b$, refer to lubricant, top, and bottom particles, respectively. The parameters of the potential (1) are chosen to be equal for all atoms, $U^{l l}=U^{t l}=U^{b l}=U_{0}=1$ and $r^{l l}=r^{t l}$ $=r^{b l}=r_{0}=1$, namely we study the tribological response in the perfectly commensurate case and in an "intermediate" interaction range with respect to those considered in Ref. 16 (neither "soft" nor "hard" lubricant). No direct interaction between the top and bottom substrates is taken into account. The cutoff radius for interactions is $r^{*}=2.5 r_{0}$.

The dynamics of the top plate with coordinates $\left(X_{\text {top }}, Y_{\text {top }}\right)$ satisfies the equations

$$
M \ddot{X}_{\text {top }}+\sum_{i=1}^{n_{l}} \sum_{j=1}^{n_{t}} \frac{d}{d x_{j}^{t}} U^{t l}\left(\left|\mathbf{r}_{i}^{l}-\mathbf{r}_{j}^{t}\right|\right)+K\left(X_{\mathrm{top}}-V_{\text {ext }} t\right)=0
$$
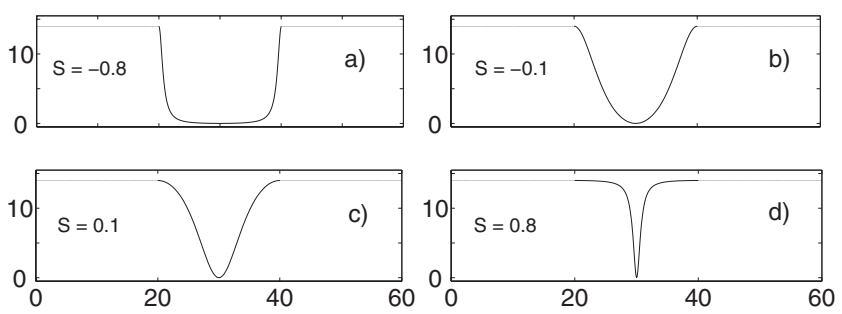

FIG. 2. Different shapes of the bottom substrate obtained for four distinct values of the parameter $s$.

$$
M \ddot{Y}_{\text {top }}+\sum_{i=1}^{n_{l}} \sum_{j=1}^{n_{t}} \frac{d}{d y_{j}^{t}} U^{t l}\left(\left|\mathbf{r}_{i}^{l}-\mathbf{r}_{j}^{t}\right|\right)+\vec{L}=0 .
$$

The equations of motion for the displacement vectors $\mathbf{r}_{i}^{l}$ of the $n_{l}$ lubricant atoms are given by

$$
\begin{aligned}
m \ddot{u}_{i} & +\frac{d}{d u_{i}} \sum_{i \neq j}^{n_{l}} U^{l l}\left(\left|\mathbf{r}_{i}^{l}-\mathbf{r}_{j}^{l}\right|\right)+\sum_{\beta=t, b} \frac{d}{d u_{i}}\left[\sum_{i}^{n_{l}} \sum_{j}^{n_{\beta}} U^{\beta l}\left(\left|\mathbf{r}_{i}^{l}-\mathbf{r}_{j}^{\beta}\right|\right)\right] \\
& + \text { N.H.C. }=0
\end{aligned}
$$

where $u_{i}=x_{i}^{l}$ or $y_{i}^{l}$. To simulate finite temperature in this driven model, we use a standard implementation of the Nosé-Hoover thermostat chain (N.H.C.), ${ }^{17,18}$ rescaling particle velocities with respect to the instantaneous lubricant center of mass velocity. The thermostat chain acts equally on all lubricant particles $j=1, \ldots n_{l}$. The equations of motion are integrated using the standard fourth-order Runge-Kutta algorithm with time step $\delta t=0.005$. All units are expressed in LJ units $U_{0}, r_{0}$ and mass $m_{0}$ of the individual atoms. We can take as reference units the interaction parameters between two Xe atoms: $U_{0}=19.83 \mathrm{meV}$ (corresponding to a temperature of $230 \mathrm{~K}), r_{0}=4.055 \AA$ and the mass $m_{0}=2.16$ $\times 10^{-22} \mathrm{~g}$, whence the unit of time is $t_{0}=\left(m_{0} r_{0}^{2} / U_{0}\right)^{1 / 2}$ $=3.345$ ps. The other units can be derived and we have for the force $F_{0}=7.8 \mathrm{pN}$, for the velocity $V_{0}=1.7 \times 10^{2} \mathrm{~m} / \mathrm{s}$. The value of elastic constant $K=7$, used in all simulations, corresponds to $1.34 \times 10^{-1} \mathrm{~N} / \mathrm{m}$.

The shape of the pattern groove in the bottom substrate is modeled by means of the Peyrard-Remoissenet function ${ }^{19}$

$$
U_{P R}(x)=\frac{d}{2} \frac{(1+s)^{2}\left(1-\cos \left(\frac{2 \pi}{w} x\right)\right)}{1+s^{2}-2 s \cos \left(\frac{2 \pi}{w} x\right)},|s|<1 .
$$

The parameter $s$ in Eq. (4) characterizes different shapes of the groove, ranging from spiky to flat, large pattern (Fig. 2), while the parameters $d$ and $w$ define its depth and width, respectively. In the present paper, we focus on the simulation results for a specific, even though not too restrictive, case $[s=0.1, w=20, d=14$ (Fig. 2(c)]. A detailed analysis of the tribological response dependence upon the pattern shape (groove geometry, depth and width) is going to be addressed in a more systematic numerical investigation.

In Fig. 1, we show the system after relaxation. At the beginning of the simulation, the groove is empty and the particles, confined between the top and bottom substrates, are ordered in layers and placed at a uniform wide separation. Due to the applied load, the lubricant particles begin to 
fill up the available void space. After a time interval $\Delta t_{1}$, although the groove is not yet completely filled, the oscillations of the top plate have been considerably damped. At this time, the stage attached to the top substrate through the spring $K$ begins to move at the constant velocity $V_{\text {ext }}$. A subsequent time interval $\Delta t_{2}$ is necessary to reach the stationary sliding state: the bottom groove is filled and three layers form between the top plate and the bottom terraces. We start then to calculate and record the system characteristics of relevant physical interest. From a computational point of view, in order to mimic reasonably the physical behavior of a stationary moving state in a boundary lubricated interface, it is important to choose a suitable number of lubricant particles in such a way that the groove fills up and an integer number of film layers (e.g., three in our case) is formed with particles uniformly distributed and no intrinsic defects. $n_{l}=290$ has been actually taken as the largest number for which the film thickness (between the top plate and the bottom terraces) remains equal to three full layers, the addition of extra particles resulting in an unstable situation with thickness irregularly oscillating between three and four layers.

In our system, the spring elongation of the driving apparatus, $\left(X_{\text {top }}(t)-V_{\text {ext }} t\right)$, gives a direct measure of the lateral force $F_{L}$ acting at time $t$ on the moving top plate. Once stationarity is achieved, we calculate the friction force $F_{\text {fric }}$ as the lateral force averaged over a sufficiently long-time interval

$$
F_{\text {fric }}=\left\langle F_{L}\right\rangle=K\left\langle X_{\text {top }}(t)-V_{\text {ext }} t\right\rangle,
$$

where \langle\rangle stands for time average. In this work, we focus on the case of commensurability among the three inherent system "lengths" $r^{l l}, r^{t l}, r^{b l}$. Experimentally, commensurability is not a common situation and can only be achieved under well-controlled operating conditions. ${ }^{20}$ However, our modeling could represent the physical situation in which two lubricant layers stick to the confining substrates and become "rigid." Commensurability means optimal structural match between the crystalline lubricant and the confining walls. This structural interlocking enhances the ordering tendency which also results from entropy reduction of the disordered molecules near the walls. ${ }^{21}$ In our case, this increased ordering tendency competes with the disorder effect due to the presence of grooves.

In order to highlight the possible effectiveness of substrate nanopatterning, aiming at improving tribological properties of mechanical sliding contacts, simulations have been done in the range of model parameters where the high dissipative stick-slip dynamics takes place. We have observed stick-slip regimes for a large interval of increasing velocities, until suppression. Thus, we focused on the behavior of the system for velocities $V_{\text {ext }}$ such that stick-slip is not suppressed but its period is sufficiently small (the faster the stage velocity, the smaller the stick-slip period). Taking care of this, the lateral force can be correctly averaged on the time interval accessible by the numerical simulation.

\section{RESULTS}

We have studied the system response by mainly varying three significant physical parameters: the load $\vec{L}$, the stage flat substrate

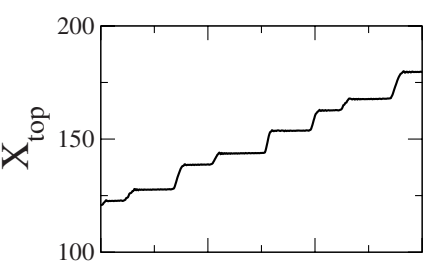

patterned substrate
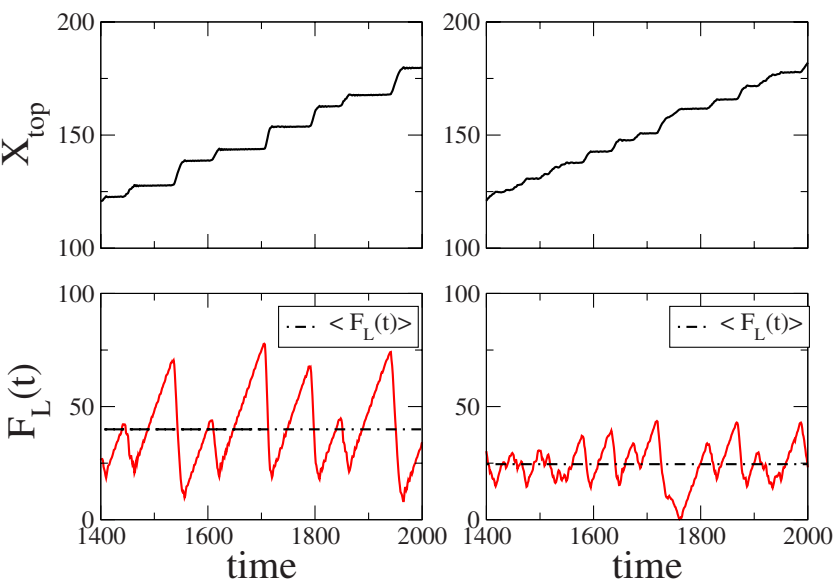

FIG. 3. (Color online) Comparison between the stick-slip behavior in the case of flat substrate (left panels) and patterned substrate (right panels). Notice the mitigation of the stick-slip regime with consequent reduction of the friction force. Here $n_{l}=290$ and 180, in the patterned and the flat case, $T=0.6, K=7, V_{\text {ext }}=0.1, L=60$. $(L-J$ units).

velocity $V_{\text {ext }}$, and the temperature $T$. This has required to perform a large number of simulations with a sufficiently small time step suitable for atomistic calculations. In presence of patterned substrates, for a large range of applied load and temperature values, we have found a substantial reduction of the tribological undesirable stick-slip regime, with a consequent drop in the sliding friction values.

Figure 3 shows the curves representing the top plate coordinate $X_{\text {top }}$ (upper panels) and the corresponding lateral force $F_{L}=K\left[X_{\text {top }}(t)-V_{\text {ext }} t\right]$ (lower panels) as a function of time in the flat (left) and patterned (right) case. The left lower panel clearly displays the typical saw-tooth dependence of $F_{L}$, the hallmark of stick-slip motion. Stick-slip as a phenomenon is quite general and extremely well known. It has been observed in many simulations of microscopic friction, as well as in the tip dynamics of atomic force microscopy and in surface force apparatus experiments of confined lubricants under shear (see, e.g., Ref. 22 and references therein). Energy is stored in the springs while atoms are trapped in a metastable state (sticking), and it is converted to kinetic energy as the atoms pop to the next metastable state (slipping). This time dependence of $F_{L}$ is reflected in the corresponding staircase temporal behavior of the top plate coordinate $X_{\text {top }}$ (left upper panel). The value of the spatial distance covered during the slip events and the regularperiodic or intermittent-chaotic temporal alternation between stick and slip can be affected by several factors: the intrinsic periodicity of the lattices defining the substrate potentials, the potential energy landscape of accessible metastable states, the specific features of imposing driving, etc. As displayed in the right panels of Fig. 3, substrate patterning leads to a mitigation of the stick-slip regime with a consequent reduction of the time-averaged lateral force $\left\langle F_{L}\right\rangle$ (dash-dotted lines in lower panels).

In Fig. 4, the friction force $F_{\text {fric }}$ is shown as a function of system temperature $T$, at fixed load $L$ and external driving 


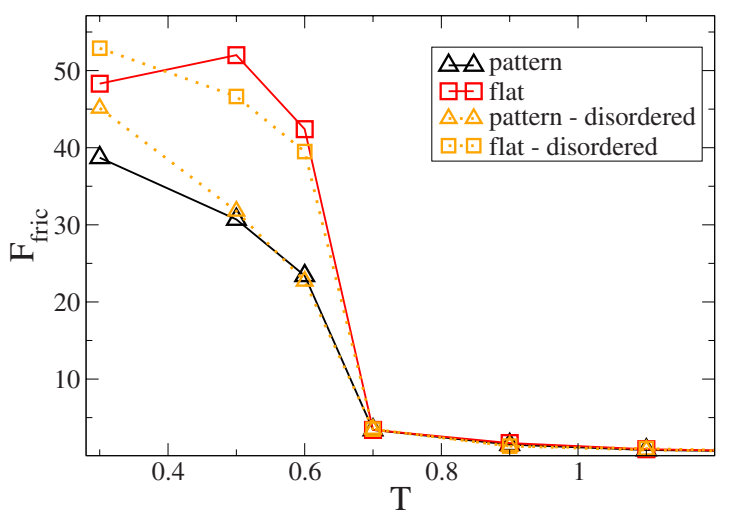

FIG. 4. (Color online) Friction force as a function of temperature for a flat (squares) and a patterned (triangles) substrate. Solid line: perfectly crystalline substrates. Dotted line: disordered substrates. There is an appreciable reduction of the friction force only below the threshold temperature $T=0.7$. For higher temperatures, the lubricant is liquid. Here $L=60, V_{\text {ext }}=0.1$.

velocity $V_{\text {ext }}$, for perfect crystalline substrates (solid curves). Friction is significantly reduced by patterning for temperatures below $T \approx 0.7$. Above $T \approx 0.7$, both in the flat and patterned case, the structure of the lubricant is typical of a hightemperature liquid, as it can be seen from the radial distribution function $g(r)$ shown in Fig. 5. In these conditions the patterning seems to be ineffective.

Weakening the commensurability constraint between lubricant and confining walls, we have proved that our numerical results hold true also in the presence of "irregular" (e.g., quenched disordered) substrates. By randomly displacing the atomic positions in the two substrates (even up to $\% 10$ of lattice equilibrium spacing), along both the horizontal and the vertical axes, we have obtained very similar qualitative results as shown by the two dotted curves in Fig. 4. Next, we have investigated the dependence of $F_{\text {fric }}$ as a function of the applied load, at fixed temperature and external velocity (Fig. 6). At low loads, the lubricant is liquid (with a radial distribution function pretty similar to that of Fig. 5); at higher loads (above $L \approx 50$ ), the confining pressure is sufficient to induce a "solidlike" phase in the film layers, making the

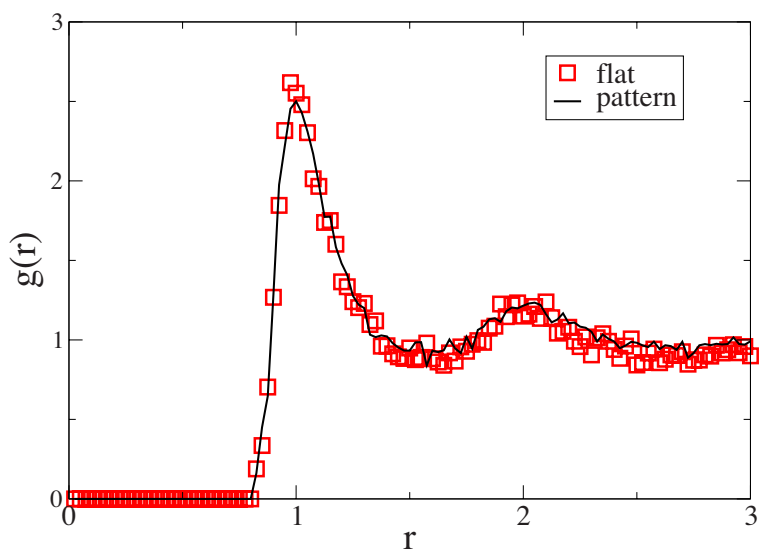

FIG. 5. (Color online) Comparison between the $g(r)$ calculated in the patterned and flat case at $T=1.1$ (above the threshold temperature $T \approx 0.7$ ), showing the typical trend of a liquid.

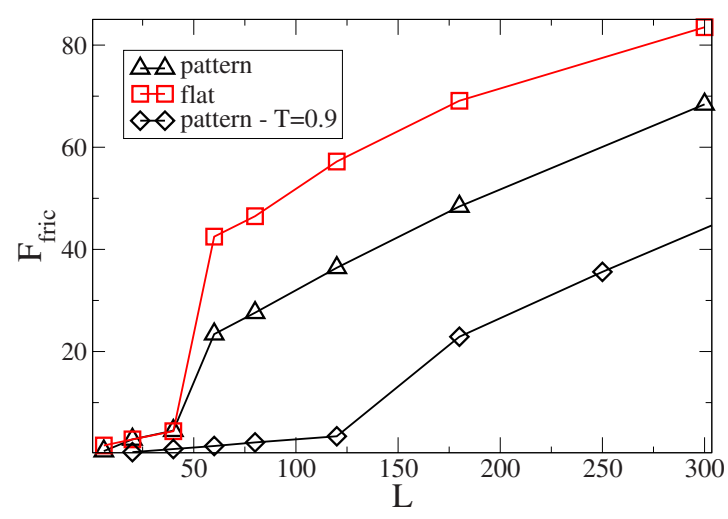

FIG. 6. (Color online) Friction as a function of load in the case of a flat (squares) and a patterned (triangles) substrate. There is an appreciable reduction of the friction force above the threshold $L$ $=40$. For lower loads, the lubricant is liquid. Here $T=0.6, V_{\text {ext }}$ $=0.1$ ( $L-J$ units $)$. The diamond curve refers to the patterned case at $T=0.9$.

nanopatterned substrate tribologically more favorable. Operating at higher values of temperature leads, as expected, to an increase in the threshold value of the loading force (diamond curve in Fig. 6). Last, the behavior of $F_{\text {fric }}$ as a function of the external stage velocity $V_{\text {ext }}$ is shown in Fig. 7, keeping the remaining physical parameters unchanged. Over the wide range of external velocities investigated, comparing the system frictional response to that for flat substrates, a significant decrease of $F_{\text {fric }}$ has always been observed in presence of nanopatterning. Besides, by increasing $V_{\text {ext }}$, the system dynamics becomes unstable for the flat geometry interface: the lubricant passes intermittently from a three to a four-layer state and vice versa. The transition from three to four layers is accompanied by an increase of the $y$ coordinate of the top plate and a decrease of friction.

We want now to investigate which are the lubricant features in the regime of "reduced friction" (for suitable applied $L, T$ and $\left.V_{\text {ext }}\right)$ due to nanopatterned substrates. Since our

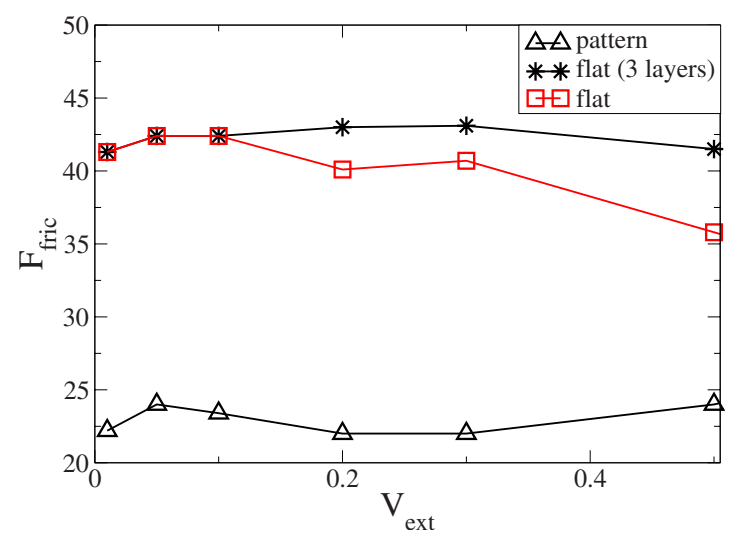

FIG. 7. (Color online) Friction as a function of $V_{\text {ext }}$ in presence of a flat (squares) and a patterned (triangles) substrate. In the flat case and for velocities above $V_{\text {ext }}=0.1$ the lubricant is unstable and passes continuously from a three to a four-layer state and vice versa. The stars represent $F_{\text {fric }}$ (in the flat case) calculated only over the time intervals where the lubricant is in the three-layer state. Here $T=0.6, L=60$ ( $L-J$ units). 


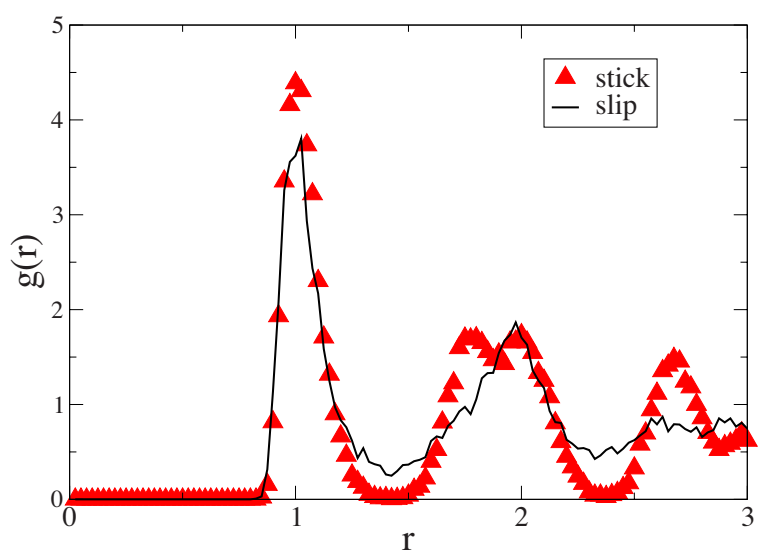

FIG. 8. (Color online) $g(r)$ calculation at $T=0.6$ in the case of flat substrate in the stick (triangles) and slip phase (solid line). In the stick phase, the peaks are typical of an hexagonal structure.

system shows an alternation of a static and dynamical phase (stick-slip), it turns out to be convenient to distinguish between the stick and slip phase. As displayed in Fig. 8 for flat substrates, in the stick phase the radial distribution function presents the typical peak features of an hexagonal solidlike structure, whereas in the slip phase $g(r)$ shows a more liquidlike profile. This behavior is related to the well-known stick-slip melting-freezing mechanism of the embedded lubricant. ${ }^{13}$ Before analyzing the $g(r)$ in the patterned case, we consider Fig. 9 which reports the trajectories of lubricant particles in the flat (upper panel) and patterned (lower panel) case over a time interval $\Delta t=200$ of the dynamics, comprising both stick and slip phases. Each point represents the position of a single particle at a given instant of time $t$. This figure clearly displays how the structure of the lubricant in the groove is much more disordered than over the terraces. At the center of the groove the hexagonal structure, typical of a solid lubricant, is lost.

Here, as shown in Fig. 10(a), the lubricant is always liquidlike, irrespective of the stick and slip phase. This disorder, even if certainly less pronounced, shows as well over the
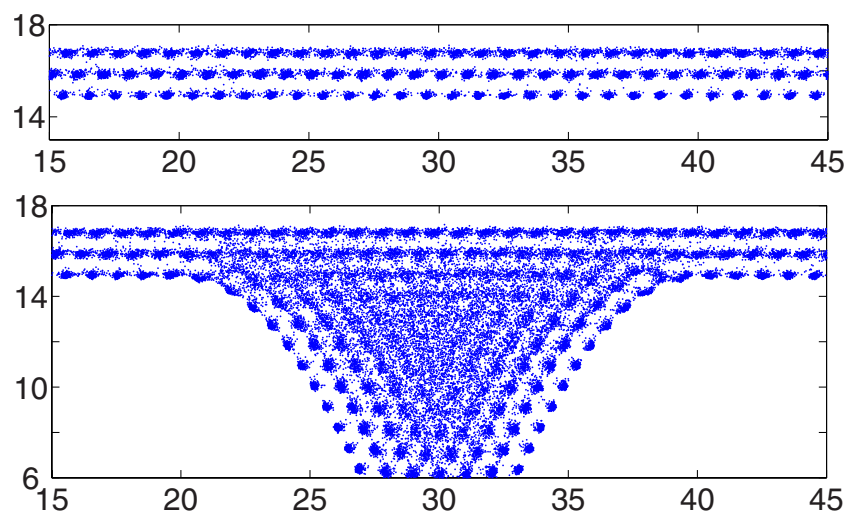

FIG. 9. (Color online) Trajectories of all lubricant particles for a time interval $\Delta t=200$. Top) Flat substrate. Bottom) Patterned substrate. The loss of the hexagonal structure at the center of the groove is clearly visible. The points between layers indicate particles passing from one layer to another. Here $T=0.6, V_{\text {ext }}=0.1, L$ $=60$ ( $L-J$ units $)$.
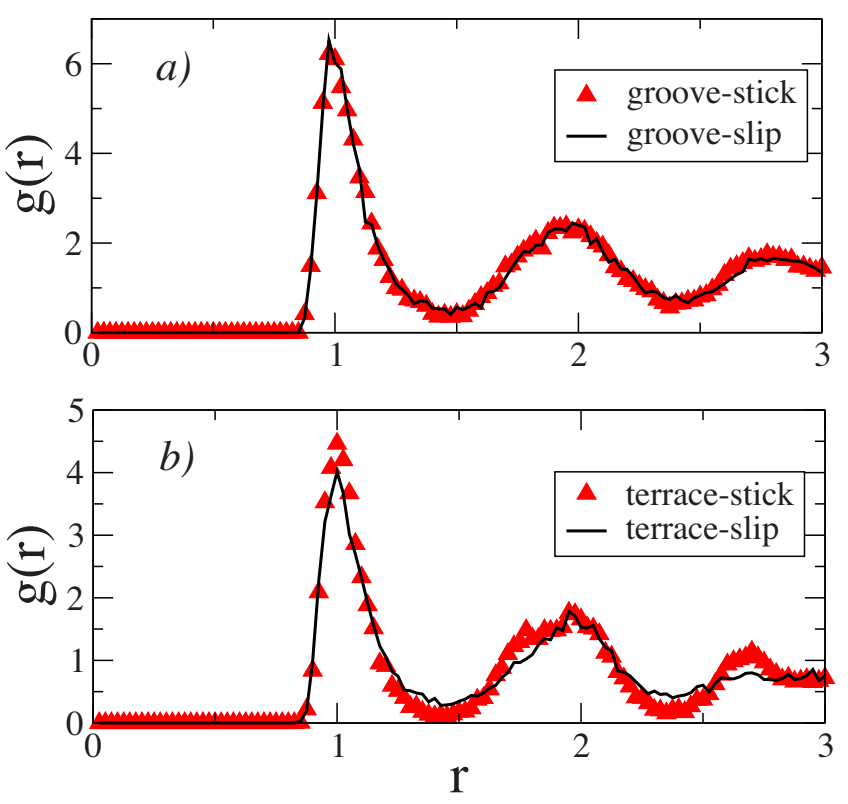

FIG. 10. (Color online) (a) $g(r)$ calculation for the particles in the groove. The behavior is liquidlike and very similar in the stick (triangles) and slip (solid line) phases. (b) $g(r)$ calculation for the particles in the terraces in the stick and slip phase. The trend indicates a 'more ordered' lubricant structure in the terraces than in the groove. Here $T=0.6, V_{\mathrm{ext}}=0.1, L=60$ ( $L-J$ units).

terraces, where we never find a solidlike structure, neither in the stick nor in the slip case [Fig. 10(b)]: even at stick the radial distribution $g(r)$ is clearly different from 0 at $r \approx 1.5$.

In literature, confinement-induced liquid-solid transitions have been reported. ${ }^{14}$ By reducing the distance between the two confining flat walls to few atomic lubricant layers, it is possible to induce layering phenomena and, often, solidification: the confinement decreases the entropy of the film and shifts the bulk melting transition to higher temperatures and lower pressures. Referring to this, in our system we could expect a solidlike (or "more ordered") phase of the thin film nearby the terraces and a liquid one in the groove, where the lubricant particles are far apart from the substrates.

A system of molecules confined between two smooth walls was previously studied ${ }^{21}$ by means of a mean-field theory for confinement-induced phase transition based on a Ginzburg-Landau expression for the free energy. A surfaceordered phase was shown to exist in presence of a large enough reduction of the surface free energy achieved by surface ordering. The phase diagram includes this additional region in which the layers adjacent to the walls are crystalline but, at the center, the ordering almost vanishes. In our model, the structural matching, due to perfect commensurability between lubricant and substrates, should enhance the surface ordering. A very limited diffusive tendency, visible in the upper panel of Fig. 9 (points between layers) for flat substrates, does not contrast with this hypothesis; experimental studies ${ }^{23}$ have in fact proved that the observed confinement-induced "solidification" does not correspond to a perfect crystallized structure but that a small diffusion is always present.

Substrate patterning destroys the horizontal ordering generated by the confining flat walls, inducing a molten zone 

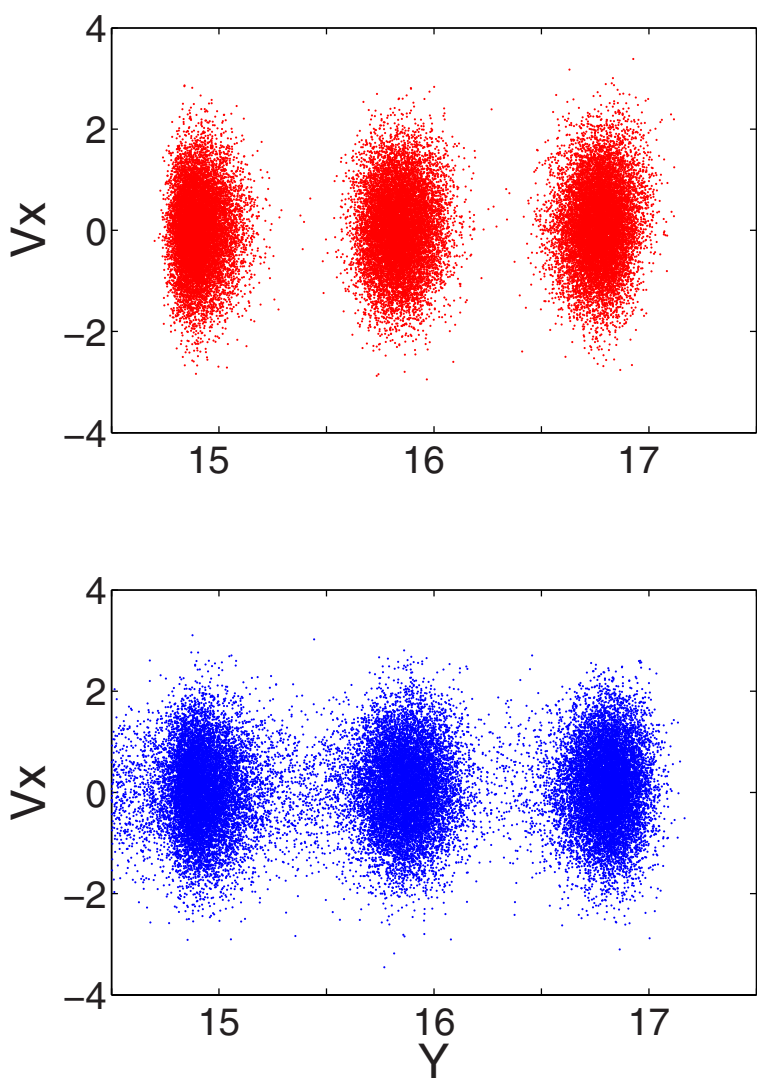

FIG. 11. (Color online) Projection of the phase space on the $\left\{V_{x}, y\right\}$ plane for a time interval $\Delta t=200$. Top) Flat substrate. Down) Patterned substrate. The points between the three spots indicate the particles that migrate from a layer to another. Here $T=0.6, V_{\text {ext }}$ $=0.1, L=60$ ( $L-J$ units $)$

nearby the grooves. However, as stated before, this patterning disorder effect extends beyond the groove itself and the radial distribution function, contrary to the flat interface (Fig. 8), never vanishes (for $r>r_{0}$ ) even over the flat terraces [Fig. 10(b)]. This is also reflected in Fig. 9 (lower panel), showing the occurrence of a visible interlayer mixing mechanism in the groove as well as over the terraces; we have noted that the diffusion turns to be particularly visible to the left of the groove, that is, shifted toward the opposite direction of the top driven motion.

To quantify this interlayer diffusion, we have also calculated $n_{p}\left(\delta t^{*}\right)$, the average number of particles that, after a given reference time $\delta t^{*}$, passes from one layer to another. We found for $\delta t^{*}=10 \times$ time-step that $\bar{n}_{p}\left(\delta t^{*}\right)=0.01$ in the flat case and 0.34 in the patterned case. The value of $\overline{n_{p}}$ strongly depends on the temperature and increases linearly with $\delta t^{*}$. The strong increase of the interlayer mixing is clearly visible from Fig. 11, representing the projection of the phase space on the $\left\{V_{x}, y\right\}$ plane in the flat (upper panel) and patterned (lower panel) case for a time interval $\Delta t=200\left(V_{x}\right.$ is the $x$ component of the velocity of the particles). The three separated spots indicate the spatial particle separation (along the vertical $y$ direction) of the three formed lubricant layers. Also in this case, the points in between represent particles migrating from one layer to another. For flat substrates, the lubricant exhibits a layer over layer sliding with strong one-

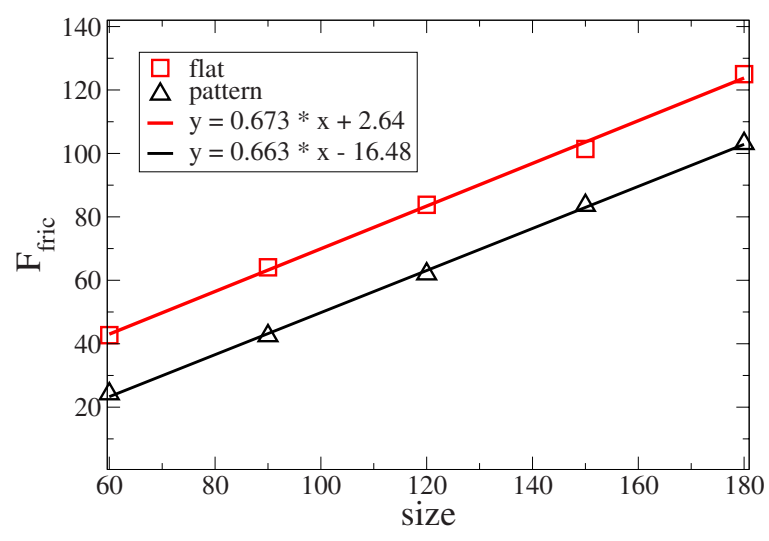

FIG. 12. (Color online) Friction as a function of the size of the system in the flat (squares) and patterned (triangles) case. The calculated values of $F_{\text {fric }}$ are all related to the same groove geometry. (Peyrard-Remoissenet geometry with $s=0.1, w=20$ and $d=14$ ). The trends are linear with the same slope. Here $T=0.6, V_{\text {ext }}=0.1, L$ $=60$ ( $L-J$ units $)$

dimensional order within the shearing layers of atoms, where each layer moves as a whole. In the patterned case, due to the increased diffusion, this layer over layer structure is not so evident. We have evaluated the layer drift velocities defining its $x$ component at time $t$ is as:

$$
V_{J, x}(t)=\frac{1}{N_{J}(t)} \sum_{i=1}^{N_{J}(t)} v_{i, x}(t)
$$

where $N_{J}(t)$ is the number of particles in the layer $J$ at time $t$ and $v_{i, x}(t)$ is the velocity $x$ component of particle $i$ in the layer $J$ at time $t$. For the flat geometry, the time-averaged layer velocities are $\left\langle V_{1, x}\right\rangle=0.0078,\left\langle V_{2, x}\right\rangle=0.042,\left\langle V_{3, x}\right\rangle$ $=0.085$, while for the patterned interface $\left\langle V_{1, x}\right\rangle=0.014$, $\left\langle V_{2, x}\right\rangle=0.062,\left\langle V_{3, x}\right\rangle=0.094$. In this last case, the velocity of the first layer (the one in contact with the immobile bottom substrate) is strongly increased. This enhanced film mobility, at the lubricant-substrate interface, favors the observed stickslip reduction shown in Fig. 3 .

\section{DENSITY OF THE GROOVES}

By varying the separation between the grooves, we have also investigated the behavior of $F_{\text {fric }}$ as a function of the pattern density. Keeping the groove shape unchanged (Peyrard-Remoissenet geometry with $s=0.1, w=20$ and $d$ $=14$ ), we have increased the size of the simulation box (size) enlarging the flat terraces at both of its sides. In particular we have analyzed systems $3 / 2,2,5 / 2$, and 3 times larger than the original one displayed in Fig. 1. The results have been compared to those for flat substrates of equal size. The simulation data displayed in the Fig. 12 have been obtained by keeping the pressure ( $\mathrm{L} /$ size $)$ constant. The plot shows a linear proportionality of $F_{\text {fric }}$ to the system size, both for the flat and the patterned substrate, highlighting a linearity dependence on the actual "contact area" (line of contact). The slope of the two lines is approximately the same, with that of the patterned case (triangles) just shifted down with respect to 
the one of the flat case (squares). As already stated, the groove creates a disturbance region in the confined lubricant, with a consequent reduction of the effective contact area responsible for the geometrical interlocking. Directly from the fitted curves of Fig. 12, it is possible to estimate the extent $l_{d}$ of the disturbance region as the distance between the two intersection points of these curves with the horizontal axis. This evaluation gives $l_{d} \simeq 29$, which is indeed larger than the width of the groove but smaller than the separation between two successive grooves; thus, in our simulations, even if no significant "interaction" occurs between successive grooves, the pattern-produced disorder does propagate also over the flat terraces.

\section{CONCLUSIONS}

The above analysis and numerical results have shown how the frictional behavior of a lubricant film in boundarylubricated regime is significantly affected by the presence of nanoscale superficial patterning of the moving confining walls. The main effect of the nanopatterning is the mitigation of the high dissipative stick-slip regime with a consequent drop of sliding friction. This mitigation is due to the local melting of the lubricant in correspondence of the groove and to an increasing interdiffusive behavior between the layers. As previously reported, ${ }^{21}$ solidification may be induced by wall confinement. The presence of patterned substrates is disadvantageous to the formation of a solidlike phase, favoring disorder and preventing possible geometrical interlocking. Weakening the commensurability constraint between lu- bricant and confining walls, we have proved that our conclusions about nanopatterning effectiveness hold true also in the presence of disordered substrates. Preliminary numerical simulations seem to show that even the presence of swelling at the substrate patterned area (typical of FIB nanofabrication ${ }^{5,6}$ ) does not affect much the qualitative picture.

Besides, the shape of the groove can play an important role in the tribological response of the sliding system. During motion, the lubricant molecules closer to the rigid walls tend to align following the shape of the walls themselves. This can create different ordering tendency and important geometrical mismatch to increase lubricant disorder and favor melting. Optimization of the pattern geometry can therefore reduce the sliding friction further, surely deserving future investigations.

\section{ACKNOWLEDGMENTS}

This research was partially supported by PRRIITT (Regione Emilia Romagna), Net-Lab "Surfaces \& Coatings for Advanced Mechanics and Nanomechanics" (SUP\&RMAN) and by the HPC-EUROPA project (R113-CT-2003-506079), with the support of the European Community-Research Infrastructure Action under the FP6 "Structuring the European Research Area" Programme. R.C. thanks all staff members at the Amsterdam Supercomputing Center SARA and Radboud University Nijmegen for kind hospitality. Discussions with O.M. Braun, G.C. Gazzadi, and S. Valeri are gratefully acknowledged.
${ }^{1}$ I. Etsion, Tribol. Lett. 17, 733 (2004).

${ }^{2}$ B. Raeymaekers, I. Etsion, and F. E. Talke, Tribol. Lett. 27, 89 (2007).

${ }^{3}$ U. Pettersson and S. Jacobson, Tribol. Lett. 17, 553 (2004).

${ }^{4}$ A. Blatter, M. Maillat, S. M. Pimenov, G. A. Shafeev, and A. V. Simakin, Tribol. Lett. 4, 237 (1998).

${ }^{5}$ A. Lugstein, B. Basnar, G. Hobler, and E. Bertagnolli, J. Appl. Phys. 92, 4037 (2002)

${ }^{6}$ D. Marchetto, A. Rota, L. Calabri, G. C. Gazzadi, C. Menozzi, and S. Valeri, Wear 265, 577 (2008).

${ }^{7}$ P. P. Pompa, L. Martiradonna, A. Della Torre, F. Della Sala, L. Manna, M. De Vittorio, F. Calabi, R. Cingolani, and R. Rinaldi, Nat. Nanotechnol. 1, 126 (2006).

${ }^{8}$ P. A. Thompson and M. O. Robbins, Science 250, 792 (1990).

${ }^{9}$ M. Urbakh, J. Klafter, D. Gourdon, and J. Israelachvili, Nature (London) 430, 525 (2004).

${ }^{10}$ C. Cottin-Bizonne, J.-L. Barrat, L. Bocquet, and E. Charlaix, Nat. Mater. 2, 237 (2003)

${ }^{11}$ J. Gao, W. D. Luedtke, and U. Landman, J. Chem. Phys. 106, 4309 (1997)

${ }^{12}$ U. Tartaglino, I. M. Sivebaek, B. N. J. Persson, and E. Tosatti, J.
Chem. Phys. 125, 014704 (2006)

${ }^{13}$ B. N. J. Persson, Sliding Friction: Physical Principles and Applications (Springer-Verlag, Berlin, 1998); Surf. Sci. Rep. 33, 85 (1999).

${ }^{14}$ J. Klein and E. Kumacheva, J. Chem. Phys. 108, 6996 (1998); E. Kumacheva and J. Klein, ibid. 108, 7010 (1998).

${ }^{15}$ O. M. Braun and M. Peyrard, Phys. Rev. E 63, 046110 (2001).

${ }^{16}$ O. M. Braun and M. Peyrard, Phys. Rev. E 68, 011506 (2003).

${ }^{17}$ D. Frenkel and B. Smit, Understanding Molecular Simulation. From Algorithms to Applications (Academic, London, 1996).

${ }^{18}$ G. J. Martyna, M. L. Klein, and M. Tuckerman, J. Chem. Phys. 97, 2635 (1992).

${ }^{19}$ M. Peyrard and M. Remoissenet, Phys. Rev. B 26, 2886 (1982).

${ }^{20}$ E. D. Smith, M. O. Robbins, and M. Cieplak, Phys. Rev. B 54 8252 (1996).

${ }^{21}$ A. Weinstein and S. A. Safran, Europhys. Lett. 42, 61 (1998).

${ }^{22}$ M. H. Müser, M. Urbakh, and M. O. Robbins, Adv. Chem. Phys. 126, 187 (2003).

${ }^{23}$ A. Mukhopadhyay, J. Zhao, S. C. Bae, and S. Granick, Phys. Rev. Lett. 89, 136103 (2002). 